\title{
Mitochondrial oxidative phosphorylation complexes exist in the sarcolemma of skeletal muscle
}

\author{
Hyun Lee ${ }^{1}$, Seung-Hyeob Kim ${ }^{1}$, Jae-Seon Lee ${ }^{2}$, Yun-Hee Yang ${ }^{3}$, Jwa-Min Nam ${ }^{3}$, Bong-Woo Kim ${ }^{1, *}$ E Young-Gyu Ko ${ }^{1, *}$ \\ ${ }^{1}$ Division of Life Sciences, Korea University, Seoul 02841, ${ }^{2}$ Department of Biomedical Sciences, College of Medicine, Inha University, \\ Incheon 22212, ${ }^{3}$ Department of Chemistry, Seoul National University, Seoul 08826, Korea
}

\begin{abstract}
Although proteomic analyses have revealed the presence of mitochondrial oxidative phosphorylation (OXPHOS) proteins in the plasma membrane, there have been no in-depth evaluations of the presence or function of OXPHOS I-V in the plasma membrane. Here, we demonstrate the in situ localization of OXPHOS I-V complexes to the sarcolemma of skeletal muscle by immunofluorescence and immunohistochemistry. A portion of the OXPHOS I-V complex proteins was not co-stained with MitoTracker but co-localized with caveolin-3 in the sarcolemma of mouse gastrocnemius. Mitochondrial matrix-facing OXPHOS complex subunits were ectopically expressed in the sarcolemma of the non-permeabilized muscle fibers and C2C12 myotubes. The sarcolemmal localization of cytochrome c was also observed from mouse gastrocnemius muscles and C2C12 myotubes, as determined by confocal and total internal resonance fluorescence (TIRF) microscopy. Based on these data, we conclude that a portion of OXPHOS complexes is localized in the sarcolemma of skeletal muscle and may have non-canonical functions. [BMB Reports 2016; 49(2): 116-121]
\end{abstract}

\section{INTRODUCTION}

Mitochondrial oxidative phosphorylation (OXPHOS) is a major source of ATP production in eukaryotic cells. OXPHOS takes place in the inner mitochondrial membrane via five OXPHOS complexes including NADH dehydrogenase (OXPHOS I), succinate dehydrogenase (OXPHOS II), ubiquinone cytochrome $\mathrm{C}$ oxidoreductase (OXPHOS III), cytochrome $c$, cytochrome oxi-

*Corresponding authors. Young-Gyu Ko, Tel: +82-2-3290-3453; Fax: +82-2-3290-4144; E-mail: ygko@korea.ac.kr, Bong-Woo Kim, Tel: +82-2-3290-3453; Fax: +82-2-3290-4144; E-mail: kbw@ seowon.ac.kr

http://dx.doi.org/10.5483/BMBRep.2016.49.2.232

Received 10 November 2015, Revised 30 November 2015, Accepted 8 December 2015

Keywords: Cytochrome c, Extracellular NADH-dependent respiration, Oxidative phosphorylation complexes, Sarcolemma, Skeletal muscle dase (OXPHOS IV) and ATP synthase (OXPHOS V). The energy obtained by electron transport from $\mathrm{NADH}$ or succinate to oxygen via the OXPHOS I-III-IV or II-III-IV supercomplex is utilized to pump protons across the inner mitochondrial membrane, creating a proton gradient. The proton gradient is ultimately used by ATP synthase (OXPHOS V) to generate ATP from ADP and $\mathrm{P}_{i}$. In addition to ATP production, the mitochondria also contribute to cellular stress responses such as autophagy and apoptosis (1). For example, cytochrome c, a key participant in ATP synthesis, is translocated from the mitochondria to the cytosol, to trigger programmed cell death after cellular exposure to different apoptotic stimuli (2).

Lipid rafts are plasma membrane subdomains that consist of cholesterol and glycosphingolipids (3). Due to the long and saturated fatty acids of glycosphingolipids and the stiff four-ring structure of cholesterol, lipid raft subdomains have more rigid property than other phospholipid compartments with short and unsaturated fatty acids in the plasma membrane (4). Thus, the lipid rafts are easily isolated from other cellular organelles based on their detergent insolubility $(3,5)$. The detergent-resistant lipid rafts contain many different receptors and their downstream signaling molecules, making the lipid rafts serve as the center for signal transduction activity (6-9).

Surprisingly, various proteomic analyses reveal that the lipid rafts also concentrate mitochondrial OXPHOS proteins in different cell lines such as HepG2 hepatocytes (10), C2C12 myotubes (11), human adipocytes (6), THP-1 monocytes $(12,13)$, RNK (natural killer) cells (14) and human umbilical vein endothelial cells (HUVEC) $(15,16)$. The observation of mitochondrial OXPHOS proteins in the plasma membrane lipid rafts has been biochemically demonstrated by different experimental approaches such as proteomics, subcellular fractionation and surface biotin-labeling in different cell lines and tissues (10, $11,17)$. However, the finding of mitochondrial proteins in the lipid rafts has also raised the possibility of mitochondrial contamination during lipid raft isolation or the possible existence of mitochondrial lipid rafts (6).

To resolve the controversy, the in situ localization of mitochondrial OXPHOS proteins in the plasma membrane must be investigated. In this study, we show that OXPHOS I-V complexes are localized in the sarcolemmal cell membrane of skeletal muscle tissue and C2C12 myotubes. Cytochrome $\mathrm{c}$ is also 
localized in the sarcolemma based on total internal resonance fluorescence (TIRF) microscopy. The sarcolemmal localization of OXPHOS complexes may provide novel insights into the non-canonical functions of OXPHOS in the sarcolemma of skeletal muscle.

\section{RESULTS}

\section{A portion of OXPHOS complexes is not localized in the mitochondria}

The existence of mitochondrial proteins in the plasma membrane has been demonstrated by different experimental approaches such as proteomics, lipid raft and plasma membrane isolation and surface biotin-labeling in different cell lines and tissues. However, there is no report describing in situ localization of the mitochondrial OXPHOS complexes in the plasma membrane. To visualize the localization of the OXPHOS complexes in the sarcolemmal cell membrane, we used different anti-OXPHOS antibodies against the NDUFB6 subunit of OXPHOS I, SDHA subunit of OXPHOS II, core I subunit of OXPHOS III, COX4I1 subunit of OXPHOS IV and ATP synthases $\alpha$ and $\beta$ for immunofluorescence. Each of these antibodies detected a single band corresponding to each OXPHOS subunit in the immunoblotting of $\mathrm{C} 2 \mathrm{C} 12$ myoblasts and myotubes (Fig. S1), suggesting that the immunofluorescent signals for OXPHOS subunits may be specific.

We carried out immunofluorescence in mouse gastrocnemius. In cross-sections and longitudinal sections (Fig. 1 and Fig. S2), a portion of immunofluorescent signals of OXPHOS subunits in the sarcolemma was not stained with MitoTracker.

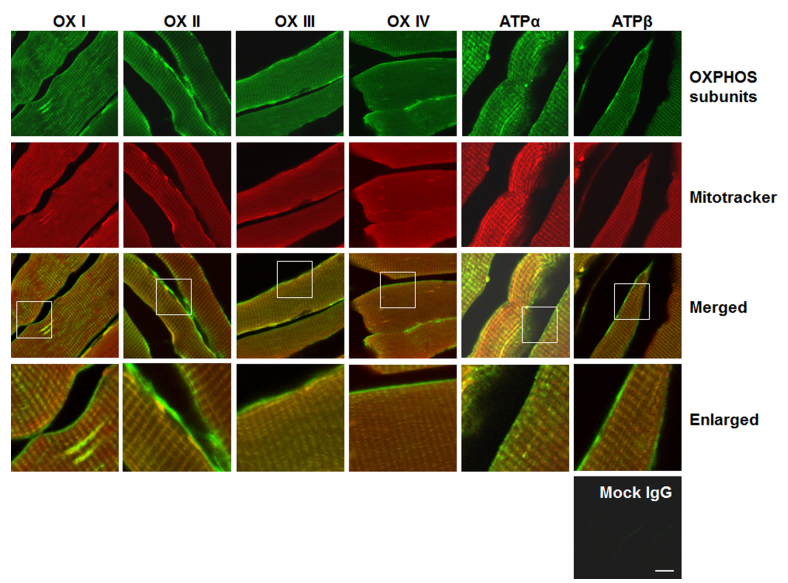

Fig. 1. A portion of OXPHOS complexes is expressed in the sarcolemma. Gastrocnemii were isolated from 8-week-old male mice. The longitudinally sectioned muscles were co-stained with MitoTracker and an anti-NDUFB6 (OX I), SDHA (OX II), core I subunit of OXPHOS III (OX III), COX4I1 (OX IV) or ATP synthase $\alpha$ or $\beta$ (ATP $\alpha$ or $\beta$ ) antibody. The boxed areas are enlarged in merged images. Scale bar $=10 \mu \mathrm{m}$.
This indicates that OXPHOS complexes in the sarcolemma were not conjugated to the mitochondria. It should be noted that there was no immunofluorescent signal in gastrocnemius tissues treated with mock IgG and secondary antibody (Fig. 1), showing that the immunofluorescent signals were specific for the indicated OXPHOS subunit.

\section{A portion of OXPHOS complexes is co-localized with caveolin-3 in the sarcolemma.}

Next, we investigated the co-localization of OXPHOS subunits with caveolin-3 in mouse gastrocnemius muscle tissue by immunofluorescence. It should be noted that caveolin-3 is specifically expressed in the sarcolemmal caveolae, which are plasma membrane invaginations formed from lipid rafts (18, 19). As shown in Fig. 2, OXPHOS subunits were co-localized with caveolin-3 in the sarcolemma, indicating that OXPHOS complexes are expressed in the sarcolemma. These fluorescent signals corresponding to the OXPHOS subunits and caveolin-3 were specific because there were no background signals in samples incubated with mock IgG.

\section{A portion of OXPHOS complexes is exposed to the cell surface}

ATP synthase is ectopically expressed on the cell surface and a portion of each OXPHOS complex is localized in the sarcolemma of skeletal muscle as shown in Figs. 1 and 2. Therefore, it is tempting to speculate that the mitochondrial matrix-facing OXPHOS subunits may also be exposed to the cell surface if the OXPHOS complexes are localized in the sarcolemma as proposed in Fig. S3. In order to address this issue, we de-

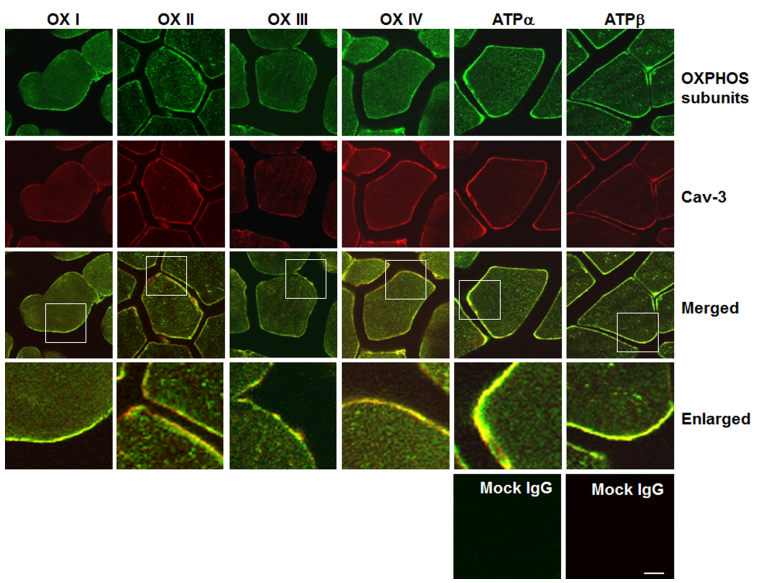

Fig. 2. A portion of OXPHOS complexes is co-localized with caveolin-3 in the sarcolemma. Gastrocnemii were isolated from 8-week-old male mice. The co-localization of caveolin-3 with each OXPHOS subunit [NDUFB6 (OX I), SDHA (OX II), core I subunit of OXPHOS III (OX III), COX4I1 (OX IV) or ATP synthase $\alpha$ or $\beta$ (ATP $\alpha$ or $\beta)]$ was determined from the cross-sectioned muscle. The boxed areas are enlarged in merged images. Scale bar $=10 \mu \mathrm{m}$. 
termined the surface expression of matrix-facing OXPHOS subunits in non-permeabilized C2C12 myotubes by immunofluorescence. For these experiments, we selected antibodies against NDUFV2 (a subunit of OXPHOS I), SDHA (a subunit of OXPHOS II), UQCR2 (a subunit of OXPHOS III), COX IV (a subunit of OXPHOS IV), and ATP_ $\beta$ (a subunit of OXPHOS V) because all of these antigens are exposed to the mitochondrial matrix. As shown in Fig. 3A, immunofluorescent signals for OXPHOS subunits were found along with the sarcolemma in non-permeabilized multinuclear myotubes. These data indicate that the matrix-facing OXPHOS subunits are also expressed on the cell surface. It should be noted that there was no fluorescent signal for cytochrome c or mock IgG (Fig. 3A). The lack of a surface signal for cytochrome $c$ suggests that cytochrome $c$ faces toward the cytosol if it localizes in the sarcolemma.

To further confirm the surface expression of each OXPHOS complex, we carried out immunofluorescence using intact muscle fibers obtained from mouse extensor digitorum longus (EDL). After collagenase treatment, the isolated muscle fibers were immediately stained with anti-OXPHOS antibodies without a permeabilization step. Fig. 3B shows strong surface expression of the OXPHOS subunits in the plasma membrane of EDL fiber. Because there was no immunofluorescent signal for cytochrome $\mathrm{C}$ and mock $\operatorname{lgG}$, the surface signals for each OXPHOS are specific.
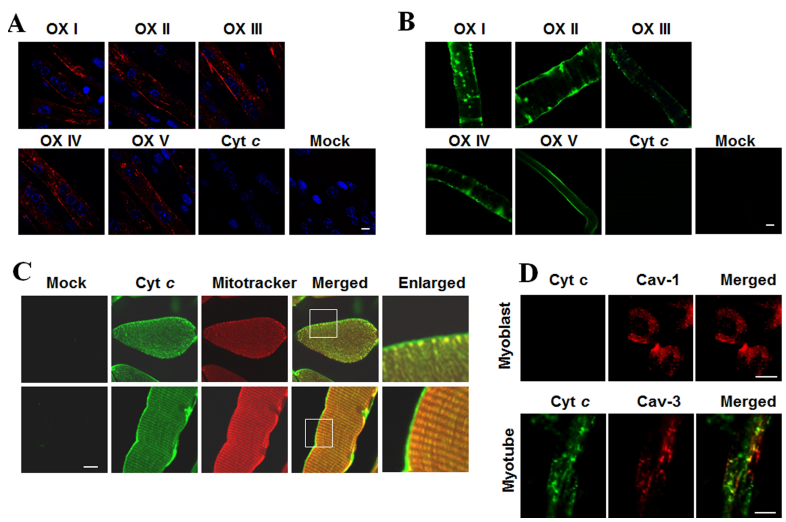

Fig. 3. OXPHOS complexes are ectopically expressed in the sarcolemma. (A) Immunofluorescence for OXPHOS proteins [NDUFV2 (OX I), SDHA (OX II), UQCR2 (OX III), COXIV (OX IV), ATP $\beta$ (OX $\mathrm{V})$ and Cytochrome $\mathrm{C}(\mathrm{Cyt} \mathrm{C})]$ was carried out using 4-day-differentiated $\mathrm{C} 2 \mathrm{C} 12$ myotubes without a permeabilization step. The nuclei were counterstained with DAPI. Scale bar $=10 \mu \mathrm{m}$. (B) Muscle fibers were isolated from extensor digitorum longus (EDL) muscles of 8-week-old mice. Immunofluorescence for OXPHOS subunits was carried out without a permeabilization step. Scale bar = $10 \mu \mathrm{m}$. (C) Cross-sectioned and longitudinally sectioned mouse gastrocnemius muscles were analyzed by immunofluorescence with an anti-cytochrome $\mathrm{C}$ antibody and MitoTracker. Scale bar $=10 \mu \mathrm{m}$. (D) Cytochrome $\mathrm{C}$ and caveolin-3 are co-localized in the sarcolemma of myotubes. Immunofluorescence of cytochrome $c$ and caveolin proteins (caveolin-1 for myoblasts and caveolin-3 for myotubes) was observed in C2C12 myoblasts and 3-day-differentiated myotubes using TIRF microscopy. Scale bar $=10 \mu \mathrm{m}$.

\section{Cytochrome $\mathrm{c}$ is localized in the sarcolemma as well as in the} mitochondria

Because all of the OXPHOS complexes were localized in the sarcolemma of skeletal muscle, as shown in Figs. 1-3, cytochrome $\mathrm{c}$ may also be localized with other OXPHOS complexes in the sarcolemma of skeletal muscle. Therefore, we monitored the cellular localization of cytochrome $c$ in mouse gastrocnemius skeletal muscles by immunohistochemistry with an anti-cytochrome $\mathrm{C}$ antibody. As shown in Fig. S4, cytochrome $c$ was highly expressed in the sarcolemma and the mitochondria isolated from the cross-sectioned and longitudinally sectioned mouse gastrocnemii. We further confirmed the sarcolemmal localization of cytochrome $\mathrm{C}$ by immunofluorescence in mouse gastrocnemius. As shown in Fig. 3C, cytochrome $\mathrm{C}$ was found in the sarcolemma that was not co-stained with MitoTracker and in the sub-sarcolemmal mitochondria that were co-stained with MitoTracker. It should be noted that incubation with mock IgG did not result in any positive fluorescent signals, indicating that the immunofluorescent signals for cytochrome $c$ are specific.

To further confirm the sarcolemmal localization of cytochrome $c$, the fluorescent signals for cytochrome $c$ and caveolin-1 or -3 (sarcolemmal marker protein of myoblasts or myotubes) were observed in $\mathrm{C} 2 \mathrm{C} 12$ myoblasts and myotubes under total internal reflection fluorescence (TIRF) microscopy which generates a high spatial resolution of fluorescent probes that are localized in the plasma membrane or just beneath the plasma membrane. As shown in Fig. 3D, the co-localization of cytochrome $\mathrm{C}$ and caveolin-3 was observed in C2C12 myotubes by TIRF microscopy, but cytochrome $\mathrm{c}$ was not co-localized with caveolin-1 in myoblasts. Thus, all these data indicate that cytochrome $c$ is localized with all of the OXPHOS complexes in the sarcolemma of skeletal muscles.

\section{Sarcolemmal OXPHOS is not involved in extracellular NADH-dependent respiration}

Based on the immunofluorescence experiments examining the OXPHOS complexes in non-permeabilized C2C12 myotubes and EDL muscle fibers, we propose the function of sarcolemmal OXPHOS complexes as shown in Fig. 4A. According to this model, electrons may transfer from extracellular NADH or succinate to oxygen via sarcolemmal OXPHOS I-III-IV or II-III-IV, generating a proton gradient across the sarcolemma. Finally, the proton gradient may be utilized to generate extracellular ATP from extracellular ADP and $\mathrm{P}_{\mathrm{i}}$ via sarcolemmal OXPHOS V. Based on this topology of sarcolemmal OXPHOS complexes, extracellular NADH may induce oxygen consumption in the sarcolemma. In order to address this hypothesis, we measured the oxygen consumption rate (OCR) of $\mathrm{C} 2 \mathrm{C} 12$ myotubes in the presence of $\mathrm{NADH}$. As shown in Fig. $4 \mathrm{~B}$, the OCR did not change after cellular exposure to $\mathrm{NADH}$, suggesting that sarcolemmal OXPHOS complexes are not involved in extracellular $\mathrm{NADH}$-dependent respiration. 
A

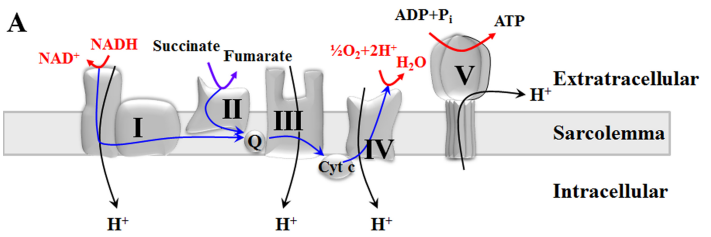

B

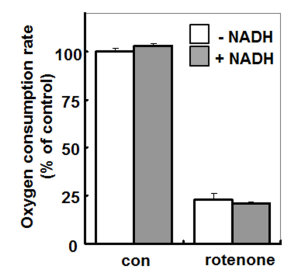

Fig. 4. Sarcolemmal OXPHOS complexes do not have the ability to consume oxygen. (A) The proposed function of the sarcolemmal OXPHOS complexes. In this model, electrons are transferred from extracellular NADH to oxygen via the sarcolemmal OXPHOS complexes, generating a proton gradient. The sarcolemmal ATP synthase generates extracellular ATP from extracellular ADP and $P_{i}$ using the proton gradient. The proton gradient is utilized by OXPHOS $\mathrm{V}$ to generate extracellular ATP from extracellular ADP and $P_{i}$. Blue lines indicate the flow of electrons and red lines indicate the flow of protons. (B) The oxygen consumption rate (OCR) was measured in 4-day-differentiated $\mathrm{C} 2 \mathrm{C} 12$ myotubes treated with different combinations of $\mathrm{NADH}(4 \mu \mathrm{M})$ and rotenone $(10 \mu \mathrm{M})$. To inhibit mitochondrial respiration, myotubes were treated with rotenone, which is an inhibitor of OXPHOS I complex.

\section{DISCUSSION}

The plasma membrane has two separate phases, the liquid-ordered phase $\left(L_{o}\right)$ and the liquid-disordered phase $\left(L_{d}\right)$. The $L_{o}$ phase, also known as lipid rafts, shows tight packing of phospholipids with longer and saturated acyl chains because of rigid cholesterol, whereas the $L_{d}$ phase contains loose packing of phospholipids with shorter and unsaturated acyl chains (6). Thus, lipid rafts of the $L_{o}$ phase cannot be solubilized by non-ionic detergents such as Triton X-100, Brij or NP40, and can be easily isolated from the $L_{d}$ phase, based on their detergent insolubility and low density in sucrose gradient ultracentrifugation. The detergent-resistant lipid rafts contain many mitochondrial and microsomal proteins as well as plasma membrane proteins based on proteomic analyses of various mammalian cells and tissues (5, 6, 10-15, 17, 20, 21). These results spark a controversy that rafts contain many contaminants originating from mitochondria and microsomes because of detergent usage (6).

However, many mitochondrial proteins, such as ATP synthase, voltage-dependent anion channels (VDAC) and prohibitin, have been unambiguously demonstrated to be localized in the plasma membrane by using various experimental approaches such as cellular fractionation, immunofluorescence, and cell surface biotinylation $(10,11,22-26)$, indicating that mitochondrial proteins isolated from the detergent-resistant lip- id rafts are not simple contaminants. Thus, we investigated the possibility that OXPHOS complexes are localized in the sarcolemma of skeletal muscle because proteomic analysis has revealed that the detergent-resistant lipid rafts isolated from C2C12 myotubes have many mitochondrial proteins, particularly, many subunits of the OXPHOS complexes (11).

All of the OXPHOS complexes and cytochrome $c$ were undoubtedly localized in the sarcolemma of skeletal muscles as determined by immunofluorescence (Figs. 1-3). The immunofluorescent analysis of skeletal muscle revealed that OXPHOS proteins are highly concentrated in the sarcolemma and the mitochondria. Because many mitochondria are also localized in sub-sarcolemmal regions based on electron microscopy (27), it is difficult to imagine that these OXPHOS complexes are localized in the subdomains of the sarcolemma that do not contain mitochondria. However, we observed that some immunofluorescent signals of OXPHOS subunits in the sarcolemma did not overlap with MitoTracker signals that have been known to specifically accumulate in the mitochondrial matrix (28), indicating that OXPHOS complexes are indeed localized in the sarcolemma of skeletal muscle.

By performing immunofluorescence experiments for OXPHOS complexes in non-permeabilized muscle fibers (Fig. $3 \mathrm{~A}, \mathrm{~B})$, we determined the topology of sarcolemmal OXPHOS complexes (Fig. 4A). According to this topology, mitochondrial matrix-facing OXPHOS subunits are exposed to the extracellular space, while mitochondrial intermembrane space-facing OXPHOS subunits are exposed to the intracellular space. Thus, a proton gradient may be generated across the sarcolemma by transfer of electrons from extracellular NADH to oxygen via the sarcolemmal OXPHOS I, III and IV complexes. Finally, the sarcolemmal OXPHOS $\vee$ may utilize the electron gradient for extracellular ATP production. Indeed, purified sarcolemma show NADH-dependent respiration activity after addition of cytochrome $c$ (11). Calzia et al. observed the OXPHOS complexes from the rod outer segment (OS), which is a continuum of the plasma membrane in the rod cells of the vertebrate retina and reported the observation of respiratory activity from the purified OS after addition of pyruvate and malate (29). However, our results show that intact $\mathrm{C} 2 \mathrm{C} 12$ myotubes did not have $\mathrm{NADH}$-dependent sarcolemmal respiration activity (Fig. 4B). In addition, the sarcolemmal ATP synthase is not involved in generating extracellular ATP because extracellular ATP generation is not disrupted in $\mathrm{C} 2 \mathrm{C} 12$ myotubes even after the knockdown of ATP synthase $\beta$ (30).

These data indicate that the sarcolemmal OXPHOS complexes are not involved in oxidative phosphorylation. Just as ectopic ATP synthase has the ability to bind to many different ligands (apolipoprotein A-I, angiostatin, $\beta$ amyloid oligomers, etc.) $(22,23,31,32)$ and to transfer HIV-1 from antigen-presenting cells to $\mathrm{CD}^{+}$cells (33), the sarcolemmal OXPHOS complexes may have as yet unknown non-canonical functions. Thus, a direction for future research on the sarcolemmal OXPHOS proteins is to identify their novel non-canonical functions. 


\section{MATERIALS AND METHODS}

\section{Animals and cell line}

C57/BL6 male mice were housed in plastic cages on a 12:12 h light-dark photoperiod with free access to water and food. Animals were handled according to the Principles of Laboratory Animal Care (NIH Publication no. 85-23, revised 1985). The mouse myoblast cell line $\mathrm{C} 2 \mathrm{C} 12$ was obtained from the American Type Culture Collection (ATCC; Manassas, VA, US) and cultured in Dulbecco's modified Eagle's medium (DMEM) supplemented with $2 \%$ penicillin/streptomycin (WelGene, Daegu, Korea) and $10 \%$ fetal bovine serum (FBS) in a $5 \% \mathrm{CO}_{2}$ incubator at $37^{\circ} \mathrm{C}$ according to the previously described method (34). In brief, C2C12 myoblasts at approximately 90-100\% confluence were differentiated into myotubes by using the growth medium with differentiation medium (DMEM supplemented with $2 \%$ penicillin/streptomycin and $2 \%$ horse serum). Every $48 \mathrm{~h}$, the differentiation medium of the myotubes was replaced with fresh medium.

\section{Antibodies and reagents}

Different anti-OXPHOS antibodies were obtained from Molecular Probes (Carlsbad, California, USA) and Santa Cruz Biotechnology (Santa Cruz, CA, USA). Anti-cytochrome C, anti-caveolin-1 and anti-caveolin-3 antibodies were obtained from BD Biosciences (Franklin Lakes, New Jersey, USA). MitoTracker Red FM dye and Alexa Fluor 488-conjugated anti-mouse IgG were purchased from Invitrogen (Carlsbad, California, USA). FITC-conjugated anti-mouse IgG and Rhodamine-conjugated anti-rabbit IgG were obtained from Abcam (Cambridge, UK). Collagenase type I and bovine serum albumin (BSA) were purchased from Sigma (St. Louis, Missouri, USA).

\section{Immunoblotting, immunofluorescence and immunohistochemistry}

The proteins in the whole cell lysates were separated on SDS-polyacrylamide gels and transferred to a PVDF membrane. The antigens were visualized by sequential treatment with specific primary antibodies, HRP-conjugated secondary antibodies, and an enhanced chemiluminescence substrate kit.

Intact muscle fibers of mouse extensor digitorum longus (EDL) muscle were isolated with $0.2 \%$ collagenase type I in DMEM and analyzed by immunofluorescence according to the previously described method (35). C2C12 myotubes were fixed with $3.7 \%$ paraformaldehyde for $10 \mathrm{~min}$, left non-permeabilized and blocked for $1 \mathrm{~h}$ with blocking buffer (5\% BSA in PBS). Subsequent incubations with primary antibodies and fluorescent-conjugated secondary antibodies, and DAPI staining were conducted at room temperature. Immunofluorescence images were captured by a laser scanning confocal microscope (ZEISS LSM 510 META, Oberkochen, Germany) or a Laser TIRF-2 system (Olympus, Tokyo, Japan).

Isolated mouse gastrocnemii were fixed with $4 \%$ paraf- ormaldehyde, snap-frozen in chilled 2-methylbutane and sectioned with a cryomicrotome. The muscle sections were subsequently incubated with blocking buffer, primary antibodies and fluorescent-conjugated secondary antibodies. Immunofluorescence images were captured by confocal microscopy.

For immunohistochemistry, mouse gastrocnemii were washed with saline and embedded in an optimal cutting temperature compound to generate frozen cross sections and longitudinal sections. Standard $6-\mu \mathrm{m}$ sections were stained using the Labeled Streptavidin Biotin kit (Dako, Produktionsvej, Glostrup, Denmark) according to the manufacturer's instructions.

\section{Measurement of oxygen consumption}

The oxygen consumption rate (OCR) was determined in $\mathrm{C} 2 \mathrm{C} 12$ myoblasts and 4-day-differentiated myotubes using a Seahorse XF24 Extracellular Flux analyzer (Seahorse Bioscience, North Billerica, MA, USA). For OCR measurement, culture media were exchanged with Dulbecco's-modified PBS (Hyclone) $1 \mathrm{~h}$ before the assay. After three baseline measurements of OCR were taken, $\mathrm{NADH}(4 \mu \mathrm{M})$ and rotenone $(10 \mu \mathrm{M})$ were separately injected into each experimental group in different combinations.

\section{ACKNOWLEDGEMENTS}

This work was supported by a grant awarded to Y.-G. Ko from the National Research Foundation (2011-0017562).

\section{REFERENCES}

1. Nunnari J and Suomalainen A (2012) Mitochondria: in sickness and in health. Cell 148, 1145-1159

2. Liu X, Kim CN, Yang J, Jemmerson R and Wang X (1996) Induction of apoptotic program in cell-free extracts: requirement for dATP and cytochrome c. Cell 86, 147-157

3. Brown DA and Rose JK (1992) Sorting of GPI-anchored proteins to glycolipid-enriched membrane subdomains during transport to the apical cell surface. Cell 68, 533-544

4. Munro S (2003) Lipid rafts: elusive or illusive? Cell 115, 377-388

5. Kim KB, Kim SI, Choo HJ et al (2004) Two-dimensional electrophoretic analysis reveals that lipid rafts are intact at physiological temperature. Proteomics 4, 3527-3535

6. Kim BW, Lee CS, Yi JS et al (2010) Lipid raft proteome reveals that oxidative phosphorylation system is associated with the plasma membrane. Expert Rev Proteomics 7, 849-866

7. Head BP, Patel HH and Insel PA (2014) Interaction of membrane/lipid rafts with the cytoskeleton: impact on signaling and function: membrane/lipid rafts, mediators of cytoskeletal arrangement and cell signaling. Biochim Biophys Acta 1838, 532-545

8. Parton RG and del Pozo MA (2013) Caveolae as plasma membrane sensors, protectors and organizers. Nat Rev Mol Cell Biol 14, 98-112

9. Whitmarsh AJ (2013) A new regulator of caveolae 
signalling. Elife 2, e01428

10. Bae TJ, Kim MS, Kim JW et al (2004) Lipid raft proteome reveals ATP synthase complex in the cell surface. Proteomics 4, 3536-3548

11. Kim BW, Lee JW, Choo HJ et al (2010) Mitochondrial oxidative phosphorylation system is recruited to detergent-resistant lipid rafts during myogenesis. Proteomics 10, 2498-2515

12. Li N, Mak A, Richards DP et al (2003) Monocyte lipid rafts contain proteins implicated in vesicular trafficking and phagosome formation. Proteomics 3, 536-548

13. Li N, Shaw AR, Zhang N, Mak A and Li L (2004) Lipid raft proteomics: analysis of in-solution digest of sodium dodecyl sulfate-solubilized lipid raft proteins by liquid chromatography-matrix-assisted laser desorption/ionization tandem mass spectrometry. Proteomics 4, 3156-3166

14. Man P, Novak P, Cebecauer M et al (2005) Mass spectrometric analysis of the glycosphingolipid-enriched microdomains of rat natural killer cells. Proteomics 5, 113-122

15. Karsan A, Blonder J, Law J et al (2005) Proteomic analysis of lipid microdomains from lipopolysaccharide-activated human endothelial cells. J Proteome Res 4, 349-357

16. Arakaki N, Nagao T, Niki R et al (2003) Possible role of cell surface $\mathrm{H}+$-ATP synthase in the extracellular ATP synthesis and proliferation of human umbilical vein endothelial cells. Mol Cancer Res 1, 931-939

17. Kim KB, Lee JW, Lee CS et al (2006) Oxidation-reduction respiratory chains and ATP synthase complex are localized in detergent-resistant lipid rafts. Proteomics 6, 2444-2453

18. Tang Z, Scherer PE, Okamoto T et al (1996) Molecular cloning of caveolin-3, a novel member of the caveolin gene family expressed predominantly in muscle. J Biol Chem 271, 2255-2261

19. Hagiwara Y, Sasaoka T, Araishi K et al (2000) Caveolin-3 deficiency causes muscle degeneration in mice. Hum Mol Genet 9, 3047-3054

20. Kim KB, Kim BW, Choo HJ et al (2009) Proteome analysis of adipocyte lipid rafts reveals that gC $1 \mathrm{qR}$ plays essential roles in adipogenesis and insulin signal transduction. Proteomics 9, 2373-2382

21. Yi JS, Mun DG, Lee $\mathrm{H}$ et al (2013) PTRF/cavin-1 is essential for multidrug resistance in cancer cells. J Proteome Res 12, 605-614

22. Moser TL, Stack MS, Asplin I, Enghild JJ, Hojrup P, Everitt L, Hubchak S, Schnaper HW and Pizzo SV (1999) Angiostatin binds ATP synthase on the surface of human endothelial cells. Proc Natl Acad Sci U S A 96, 2811-2816
23. Martinez LO, Jacquet S, Esteve JP et al (2003) Ectopic beta-chain of ATP synthase is an apolipoprotein A-I receptor in hepatic HDL endocytosis. Nature 421, 75-79

24. Terashima M, Kim KM, Adachi T et al (1994) The IgM antigen receptor of $\mathrm{B}$ lymphocytes is associated with prohibitin and a prohibitin-related protein. EMBO J 13, 3782-3792

25. Bathori G, Parolini I, Tombola F et al (1999) Porin is present in the plasma membrane where it is concentrated in caveolae and caveolae-related domains. J Biol Chem 274, 29607-29612

26. Kim BW, Choo HJ, Lee JW, Kim JH and Ko YG (2004) Extracellular ATP is generated by ATP synthase complex in adipocyte lipid rafts. Exp Mol Med 36, 476-485

27. Shaw CS, Jones DA and Wagenmakers AJ (2008) Network distribution of mitochondria and lipid droplets in human muscle fibres. Histochem Cell Biol 129, 65-72

28. Morales-Sainz L, Escobar-Ramirez A, Cruz-Torres V et al (2008) The polypeptides COX2A and COX2B are essential components of the mitochondrial cytochrome c oxidase of Toxoplasma gondii. Biochim Biophys Acta 1777, 202-210

29. Calzia D, Barabino S, Bianchini $P$ et al (2013) New findings in ATP supply in rod outer segments: insights for retinopathies. Biol Cell 105, 345-358

30. Choo HJ, Kim BW, Kwon OB, Lee CS, Choi JS and Ko YC (2008) Secretion of adenylate kinase 1 is required for extracellular ATP synthesis in $\mathrm{C} 2 \mathrm{C} 12$ myotubes. Exp Mol Med 40, 220-228

31. Schmidt C, Lepsverdize E, Chi SL et al (2008) Amyloid precursor protein and amyloid beta-peptide bind to ATP synthase and regulate its activity at the surface of neural cells. Mol Psychiatry 13, 953-969

32. Xing SL, Chen B, Shen DZ and Zhu CQ (2012) beta-amyloid peptide binds and regulates ectopic ATP synthase alpha-chain on neural surface. Int J Neurosci 122, 290-297

33. Yavlovich A, Viard M, Zhou M, Veenstra TD, Wang JM, Gong W, Heldman E, Blumenthal R and Raviv Y (2012) Ectopic ATP synthase facilitates transfer of HIV-1 from antigen-presenting cells to CD4(+) target cells. Blood 120, 1246-1253

34. Hong J, Kim BW, Choo HJ et al (2014) Mitochondrial complex I deficiency enhances skeletal myogenesis but impairs insulin signaling through SIRT1 inactivation. J Biol Chem 289, 20012-20025

35. Pasut A, Jones AE and Rudnicki MA (2013) Isolation and culture of individual myofibers and their satellite cells from adult skeletal muscle. J Vis Exp 22, e50074 